\title{
Dye chromoendoscopy leads to a higher adenoma detection in the duodenum and stomach in patients with familial adenomatous polyposis
}

\section{(ㄷ)(우우}

Authors

R. Hüneburg1,2, D. Heling ${ }^{1,2}$, D. J. Kaczmarek ${ }^{1}$, P. van Heteren ${ }^{1,2}$, M. Olthaus ${ }^{1,2}$, R. Fimmers ${ }^{3}$, M. Berger ${ }^{3}$, C. Coch ${ }^{4}$, J. F. Lau ${ }^{2,5}$, G. Kristiansen ${ }^{2,5}$, T. J. Weismüller ${ }^{1}$, I. Spier ${ }^{2,6}$, S. Aretz ${ }^{2,6}$, C. P. Strassburg ${ }^{*, 1,2}$, J. Nattermann ${ }^{*, 1,2}$

Institutions

1 Department of Internal Medicine I, University Hospital Bonn, Germany

2 National Center for Hereditary Tumor Syndromes, University Hospital Bonn, Germany

3 Institute for Medical Biometry, Informatics and Epidemiology (IMBIE), University Hospital Bonn, Germany

4 Clinical study core unit (SZB), University Hospital Bonn, Germany

5 Institute of Pathology, University Hospital Bonn, Germany

6 Institute of Human Genetics, University Hospital Bonn, Germany

submitted 24.4 .2020

accepted after revision 9.6.2020

Bibliography

Endoscopy International Open 2020; 08: E1308-E1314

DOI 10.1055/a-1220-6699

ISSN 2364-3722

(C) 2020. The Author(s).

This is an open access article published by Thieme under the terms of the Creative Commons Attribution-NonDerivative-NonCommercial License, permitting copying and reproduction so long as the original work is given appropriate credit. Contents may not be used for commecial purposes, or adapted, remixed, transformed or built upon. (https://creativecommons.org/licenses/by-nc-nd/4.0/)

Corresponding author

Robert Hüneburg, MD, Department of Internal Medicine I, University of Bonn, Venusberg-Campus 1, D-53127 Bonn, Germany

Fax: +49-228-287-19763

robert.hueneburg@ukbonn.de

\section{ABSTRACT}

Backround and study aims Duodenal cancer is the cancer most often seen in patients with familial adenomatous polyposis (FAP) who have undergone risk-reducing colonic surgery. Almost all patients with FAP eventually develop duodenal adenomas and risk for duodenal cancer is up to $12 \%$ with poor prognosis. In addition, there is a rising concern regarding increased gastric cancer risk in patients with FAP. Our aim was to enhance polyp detection by using CE (CE) with the application of indigo carmine dye.

Patient and methods We conducted a prospective, blinded study of patients with FAP undergoing endoscopic examination of the upper gastrointestinal tract. First, a standard white-light examination (WLE) was done followed by an examination performed by an endoscopist who was blinded to the previous examination, using chromoendoscopy (CE) ( $0.4 \%$ indigo carmine dye).

Results Fifty patients were included in the study. Using WLE, a median number of 13 adenomas (range 0-90) was detected compared to 23 adenomas/patient (range 0$150 ; P<0.0001)$ detected after staining, leading to a higher Spigelman stage in 16 patients $(32 \% ; P=0.0003)$. CE detected significantly more larger adenomas $(>10 \mathrm{~mm})$ than WLE (12 vs. $19 ; P=0.0391)$. In the gastric antral region, a median number of 0 adenomas (range $0-6$ ) before and 0.5 adenomas (range $0-7)$ after staining $(P=0.0025)$ were detected.

Conclusion This prospective endoscopic trial, to our knowledge the largest in patients with FAP, showed a significant impact of CE on adenoma detection and therapeutic management in the upper gastrointestinal tract. This leads to more intensive surveillance intervals.

\section{Introduction}

Familial adenomatous polyposis (FAP) is a rare, autosomal dominant, systemic disorder with a prevalence of approximate-

* These authors contributed equally. ly 1:10000 live births caused by a germline mutation in the Ade- 
nomatous polyposis coli (APC) gene [1]. FAP is characterized by development of adenomatous polyps especially in the colon, with a $100 \%$ risk of colorectal cancer by age 35 to 40 years in untreated patients [2]. In view of a myriad of colonic lesions, an effective endoscopic surveillance to prevent disease progression and development of colorectal cancer is not feasible. Therefore, risk-reducing prophylactic surgery, performed as proctocolectomy with either ileal pouch-anal anastomosis (IPAA) or reconstruction via ileorectal anastomosis (IRA), is recommended in patients with FAP.

In addition to the manifestation in the colon, most patients with FAP also have involvement in the upper gastrointestinal tract. This applies, in particular, to the duodenum with a cumulative risk of developing duodenal adenomas of up to $90 \%$ [3] and a risk of developing duodenal cancer of up to $12 \%$ [4]. Accordingly, duodenal cancer is a major cause of death in patients with FAP after prophylactic proctocolectomy [5]. Moreover, data indicated an increase in incidence of gastric cancer in patients with FAP in recent years [6].

Therefore, regular screening of the upper gastrointestinal tract is recommended in patients with FAP, although the optimal surveillance strategy has not yet been established [7-9]. Currently, surveillance intervals as well as therapeutic decisions regarding endoscopic versus surgical care are based on the Spigelman scoring system for duodenal polyposis, which was developed to allow assessment of risk of developing duodenal carcinoma ( $\triangleright$ Table 1 ) $[4,5,10]$.

Patients with FAP with a duodenal polyposis Spigelman IV have a significantly higher risk for developing duodenal cancer (up to $46 \%$ within 10 years) compared to patients with a Spigelman 0 -III situation $[3,11,12]$. However, in up to $40 \%$ of duodenal cancer cases in FAP, patients never had Spigelman IV disease $[3,11,12]$.

In view of these data, there are intensive efforts to optimize endoscopic surveillance of patients with FAP to prevent development of intestinal malignancies more effectively or to detect them at least as early as possible.

Several studies have evaluated the effect of conventional chromoendoscopy (CE) and virtual CE in patients with FAP [13-15]. However, the significance of these studies is somewhat limited due to small sample size, heterogeneous study populations, or shortcomings in study design.

The main outcome of the current study was to compare adenoma detection rates (ADRs) in the duodenum and gastric antral region using standard white-light endoscopy (WLE) versus CE in the upper gastrointestinal tract of patients with FAP. Secondary outcomes were adenoma number, size and impact of dye-spray with indigo carmine on Spigelman stage.

\section{Patients and methods}

\section{Study design}

The study was approved by the local institutional review board, the Bundesinstitut für Arzneimittel und Medizinprodukte (BfArM), and was registered at the European Clinical Trials Database (EudraCT) (EudraCT Nr.: 2014-003546-27).
- Table 1 Modified Spigelman classification for duodenal polyposis.

\begin{tabular}{|l|l|l|l|}
\hline & $\mathbf{1}$ point & $\mathbf{2}$ points & $\mathbf{3}$ points \\
\hline Polyp number & $1-4$ & $5-20$ & $>20$ \\
\hline Polyp size $(\mathrm{mm})$ & $1-4$ & $5-10$ & $>10$ \\
\hline Histology & Tubular & Tubulovillous & Villous \\
\hline Dysplasia & Low-grade & & High-grade \\
\hline
\end{tabular}

Stage $0=0$ points; stage $\mathrm{I}=1-4$ points; stage $I I=5-6$ points; stage $I I I=7-8$ points; stage IV=9-12 points

This was a prospective, blinded study conducted at a tertiary referral center (Department of Internal Medicine I, University Hospital Bonn, Germany), comparing standard WLE endoscopy and CE using indigo carmine for adenoma detection in patients with known FAP.

\section{Study population}

Patients scheduled for regular surveillance endoscopy were prospectively recruited at the National Center for Hereditary Tumor Syndromes (nzet.de) at the University Hospital Bonn between December 2016 and December 2017. Written informed consent was obtained from all subjects. Eligibility for this study was a proven diagnosis of FAP by having a pathogenic APC gene variant, age $\geq 18$ years, and ability to give an informed consent. Exclusion criteria were previous upper gastrointestinal tract surgery (Whipple procedure, pancreas-sparing duodenectomy), known allergy to indigo carmine, known coagulopathy, or American Society of Anesthesiologists (ASA) class III or higher.

Patient characteristics documented included demographics (age, gender), mutation, FAP history and previous surgeries.

\section{Endoscopy protocol}

Endoscopists and equipment: All procedures were performed by two skilled endoscopists experienced in $\mathrm{CE}(\mathrm{RH}, \mathrm{JN}, \mathrm{PVH}$, DK, TW) who were assigned to be the first or second examiner in an alternating fashion.

All endoscopes used in the study were high-resolution video endoscopes (EG-590WR, Fujifilm Germany Inc.).

Procedures were performed under conscious sedation with propofol \pm midazolam. Antispasmodic medication (butylscopolamine bromide) was given during endoscopy at the discretion of the endoscopist.

The forward-viewing endoscope was advanced until the duodenojejunal junction was reached. The duodenum was divided into three different parts: (duodenojejunal junction till periampullary region (D3), periampullary region till duodenal bulb (D2), and duodenal bulb (D1)). All polyps were evaluated and their number and sizes in each segment were documented. Polyp size was estimated using an open biopsy forceps (Endoflex CE 0483), with an open diameter of $8 \mathrm{~mm}$. The gastric antral region was also carefully inspected and detected polyps were recorded. Documentation was done by an independent observer on a specific care-report form. If the ampulla could 
not be visualized properly, a side-viewing duodenoscope was used.

Either biopsies nor polypectomies were performed during the first examination.

The following examination was done by a second endoscopist blinded to the previous examination. Again, the forward-viewing endoscope was advanced until the duodenojejunal junction was reached. A spray catheter (PW-205V, Olympus Japan) was inserted through the working channel of the endoscope. Indigo carmine dye $0.4 \%$ (Amino AG, Switzerland) was sprayed on the mucosa in a homogeneous fashion. The residual dye was suctioned away. After sufficient coating of the duodenal mucosa, all polyps were documented in terms of number, size, and localization. The gastric antral region was also carefully inspected after application of indigo carmine and detected polyps were documented. Biopsy samples were not taken until staining and counting had taken place. Histopathological sampling of at least three polyps per segment and all polyps $>1 \mathrm{~cm}$ was performed.

Examination times were documented by an independent observer. Withdrawal time was defined as the time for application of indigo carmine dye and inspection from the duodenojejunal junction to the gastric antral region without performing any endoscopic intervention.

All biopsies and endoscopic mucosal resection specimens were evaluated by two expert gastrointestinal pathologists (JL and GK), who graded dysplasia according to the Vienna classification (high grade/ low grade) [16].

Patients were contacted $7( \pm 4)$ days after examination to assess possible adverse events.

For all patients, the modified Spigelman ( $>$ Table 1 ) stage and score were assessed before and after application of indigo carmine, respectively [10]. The following criteria were used: number of adenomas, largest size, most advanced histology, and most advanced grade of dysplasia.

\section{Outcomes}

The primary outcome was ADR, defined as the fraction of patients in whom at least one adenoma was detected. Secondary endpoints were number of adenomas, size of the largest detected adenoma, Spigelman score as well as withdrawal time.

Histological findings from biopsies taken during CE were used to determine the Spigelman score, respectively, as according to the study protocol no biopsy was taken during the first examination.

\section{Statistical analysis}

Statistical analyses were performed using SAS (SAS Proprietary Software 9.4). Categorical variables were described by absolute and relative frequencies; continuous variables were described using median and range. In $>$ Table 2 , mean and standard deviation were used.

ADRs were compared with McNemars Test. The number of upper gastrointestinal tract adenomas were compared using Wilcoxon rank-sum tests. Significance level for the test of primary and secondary outcomes was set to $5 \%$ (two-sided).
- Table 2 Patient, disease, and procedure characteristics $(n=50)$.

\begin{tabular}{|l|l|}
\hline Patient and disease characteristics & \\
\hline Age (years) & \\
\hline Male gender (\%) & $40.10 \pm 13.24(19-69)$ \\
\hline Intact colon (\%) & $26(52.0)$ \\
\hline Previous risk-reducing surgery & $13(26.0)$ \\
\hline History of colectomy (\%) & $37(74.0)$ \\
\hline History of proctocolectomy (\%) & $8(21.62)$ \\
\hline Age at risk-reducing surgery (years) & $29(78.38)$ \\
\hline ' (mean \pm SD), (min,max) & $32.38 \pm 9.43(18-56)$ \\
\hline
\end{tabular}

\section{Sample size}

A sample size of 50 paired observations was found to have an $80 \%$ power to determine a difference in detection of adenomas in $20 \%$ of all cases (coincidence of the results in $80 \%$ of all cases). With an expected dropout rate of $5 \%, 53$ patients needed to be included in our study.

\section{Results}

Between December 2016 and December 2017, a total of 53 patients with known pathogenic APC mutation were enrolled into this study. All patients underwent an upper gastrointestinal examination. One patient was excluded from the final analysis because the database was closed; two patients were excluded due to inconsistent documentation. None of the patients were on any pharmacological treatment for their duodenal or colonic polyp burden. Patient characteristics are shown in > Table 2.

Fifty patients were included in the final analysis, including 24 women and 26 men with a mean age of 40 years (range 19-69). In 37 patients, a risk-reducing surgery of the lower gastrointestinal tract had been performed in the past at a mean age of 32 years (range 18-56).

Regarding the primary outcome, we observed a slightly higher ADR using CE compared to WLE (48 patients (96\%) vs. 43 patients $(86 \%) ; P=0.218)$. However, this difference failed to achieve statistical significance.

\section{Number and size of duodenal adenomas}

Before staining, a median number of 13.5 adenomas (range $0-$ 90; total 1001 adenomas) were detected. After staining, the median number of adenomas detected was 23 per patient (range 0-150; total 1719; $P<0.0001$ ) ( $\mathbf{F i g . ~} 1 \mathrm{a}$ and $>$ Table 3 ).

Additional duodenal adenomas were detected in 42 patients ( $84 \%)$, with a median number of additional adenomas detected per patient of 12.5 (total 776, range 1-87). In a subanalysis of the individual segments of the duodenum, this increase in detected adenomas could be confirmed for all investigated duodenal (D1-3) regions, although the differences were statistically significant only for D1 and D2. 


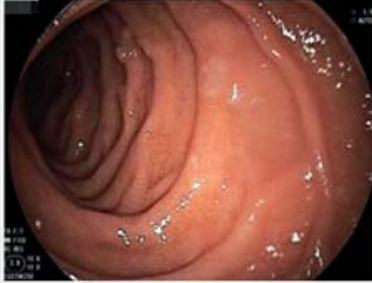

Duodenal mucosa prior stainig

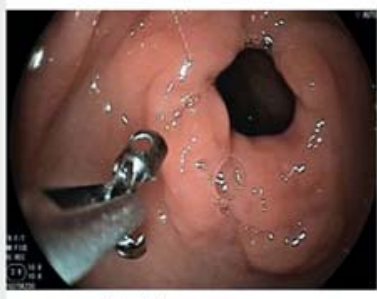

Gastric mucosa b

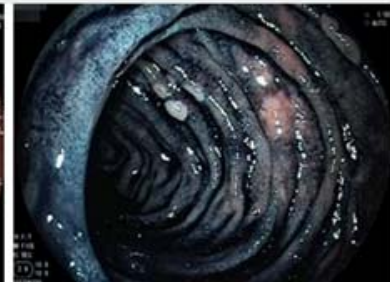

Duodenal mucosa after staining

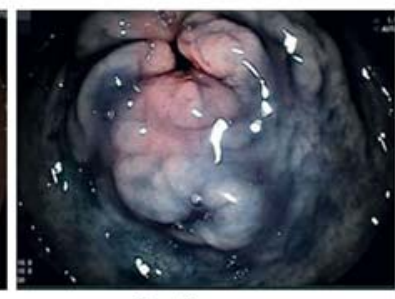

Gastric mucosa after staining
- Fig. 1 Examples of duodenal and gastric mucosa before and after staining with indigo carmine.

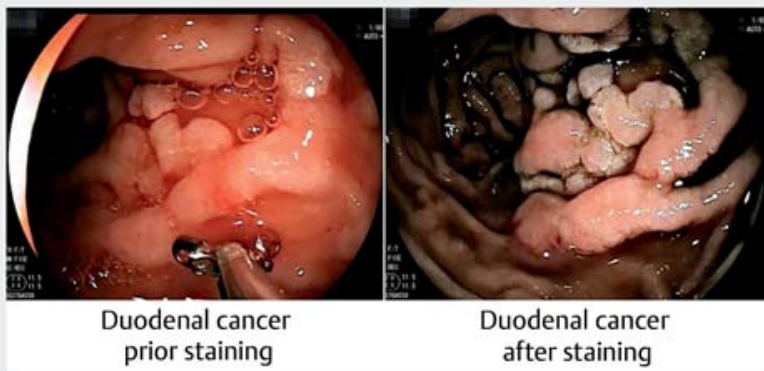

- Fig. 2 Macroscopic appearance of duodenal cancer before and after staining with indigo carmine.

According to the Spigelman staging system, adenomas were classified to be either 1 to $4 \mathrm{~mm}$ large, 5 to $10 \mathrm{~mm}$, or larger than $10 \mathrm{~mm}$, respectively. After staining, adenomas larger than $10 \mathrm{~mm}$ were detected in significantly more patients $(12$ vs. 19, $P=0.0391$; $>$ Table 3 ). In eight patients, adenomas were measured to be $>10 \mathrm{~mm}$ using CE compared to WLE. In three of these patients, WLE only detected adenomas with a size of 1 to $4 \mathrm{~mm}$, in five patients with a size of 5 to $10 \mathrm{~mm}$. In one patient, adenomas larger than $10 \mathrm{~mm}$ were detected using WLE, but measured to be 5 to $10 \mathrm{~mm}$ using $\mathrm{CE}$.

All detected adenomas were tubular adenomas with lowgrade dysplasia, except in four patients that displayed tubulovillous adenomas with low-grade dysplasia.

\section{Spigelman stage}

Using WLE, the Spigelman classification was stage 0 in six patients $(12 \%)$, stage I in nine patients $(18 \%)$, stage II in 15 patients (30\%), stage III in 17 patients (34\%) and stage IV in
- Table 3 Duodenal adenoma characteristics using white-light endoscopy (WLE) and CE (CE).

\begin{tabular}{|c|c|c|c|}
\hline Duodenal findings & WLE & CE & $P$ value \\
\hline $\begin{array}{l}\text { Median number of } \\
\text { adenomas }\end{array}$ & $13(0-90)^{1}$ & $23(0-150)^{1}$ & $<0.0001$ \\
\hline $\begin{array}{l}\text { Largest size of adeno- } \\
\text { mas }\end{array}$ & & & 0.0129 \\
\hline - $1-4 \mathrm{~mm}$ & 18 & 12 & 0.0703 \\
\hline - $5-10 \mathrm{~mm}$ & 13 & 12 & 1.000 \\
\hline - $>10 \mathrm{~mm}$ & 12 & 19 & 0.0391 \\
\hline Spigelman stage & $2(0-I V)$ & $3(0-I V)$ & 0.0003 \\
\hline \multicolumn{4}{|l|}{ D3 } \\
\hline $\begin{array}{l}\text { Median number of } \\
\text { adenomas }\end{array}$ & $6.5(0-55)$ & $10(0-50)$ & 0.0965 \\
\hline . $1-4 \mathrm{~mm}$ & 27 & 22 & 0.2266 \\
\hline - 5-10mm & 5 & 12 & 0.0654 \\
\hline . $>10 \mathrm{~mm}$ & 8 & 6 & 0.7266 \\
\hline \multicolumn{4}{|l|}{ D2 } \\
\hline $\begin{array}{l}\text { Median Number of } \\
\text { adenomas }\end{array}$ & $3(0-27)$ & $8(0-50)$ & $<0.0001$ \\
\hline $\begin{array}{l}\text { Largest size of adeno- } \\
\text { mas }\end{array}$ & & & 0.0636 \\
\hline - $1-4 \mathrm{~mm}$ & 21 & 17 & 0.3877 \\
\hline . $5-10 \mathrm{~mm}$ & 11 & 7 & 0.4240 \\
\hline . $>10 \mathrm{~mm}$ & 6 & 14 & 0.0386 \\
\hline \multicolumn{4}{|l|}{ D1 } \\
\hline $\begin{array}{l}\text { Median number of } \\
\text { adenomas }\end{array}$ & $0(0-19)$ & $4(0-50)$ & $<0.0001$ \\
\hline $\begin{array}{l}\text { Largest size of adeno- } \\
\text { mas }\end{array}$ & & & 0.1796 \\
\hline . $1-4 \mathrm{~mm}$ & 11 & 6 & 0.2266 \\
\hline - $5-10 \mathrm{~mm}$ & 6 & 4 & 0.7266 \\
\hline - $>10 \mathrm{~mm}$ & 2 & 9 & 0.0391 \\
\hline
\end{tabular}

WLE, white-light endoscopy; CE, chromoendoscopy

${ }^{1}$ In 5 patients more adenomas (all size $1-4 \mathrm{~mm}$ ) were detected in the first examination with a median number of 4 (total 20 , range $1-7$ ).

three patients (6\%). Using CE, we detected stage 0 in two patients ( $4 \%$ ), stage I in 10 patients (20\%), stage II in 10 patients (20\%), stage III in 24 patients $(48 \%)$ and stage IV in four patients ( $8 \%)$. Use of CE led to an increase in median Spigelman stage from II (range 0-IV) to III (range 0 -IV), corresponding to higher classification in 16 patients (from 0 to $>1: n=4$; from 0 to $>2: \mathrm{n}=1$; from 1 to $>2: \mathrm{n}=2$; from 2 to $>3: \mathrm{n}=8$; from 3 to $>4$ : $\mathrm{n}=1 ; P=0.0003$ ). In one patient the detection of a larger polyp, in four patients a higher number of discovered adenomas, and in 11 patients a combination of both parameters was cause for the upgrade. 
Table 4 Gastric adenoma characteristics using white-light endoscopy (WLE) and CE (CE).

\begin{tabular}{|l|c|c|c|}
\hline Gastric findings & WLE & CE & P value \\
\hline $\begin{array}{l}\text { Median number of antral } \\
\text { adenomas }\end{array}$ & $0(0-6)^{1}$ & $0.5(0-7)^{1}$ & 0.0025 \\
\hline $\begin{array}{l}\text { Largest size of antral ade- } \\
\text { nomas }\end{array}$ & 5 & 3 & 0.4531 \\
\hline $1-4 \mathrm{~mm}$ & 5 & 6 & 0.6875 \\
\hline $5-10 \mathrm{~mm}$ & 2 & 3 & 1.0000 \\
\hline$>10 \mathrm{~mm}$ & & 1.0000 \\
\hline $\begin{array}{l}\text { WLE, white-light endoscopy; CE, chromoendoscopy } \\
1 \text { In } 3 \text { patients more adenomas (all size 1-4 mm) were detected in the first } \\
\text { examination with a median number of 4 (total 9, range 1-4). }\end{array}$ \\
\hline
\end{tabular}

In a patient in whom CE led to an upgrade in Spigelman stage from III to IV, a large adenoma with macroscopic worrisome features was detected with both endoscopic methods ( Fig.2). Endoscopic biopsy revealed tubulo-villous adenoma with low-grade dysplasia. In line with current recommendations, the patient, therefore, underwent prophylactic pancreas-sparing duodenectomy. Histological examination of the resectate, however, revealed a T3N0 duodenal cancer, underpinning the potential problem of sampling errors in large duodenal adenomas.

\section{Gastric findings}

In the gastric antral region, we detected significantly more adenomas using $C E$ with a median number of 0 adenomas (range $0-6$; total number of 39 adenomas) prior staining and a median number of 0.5 adenomas (range $0-7$; total number of 61 adenomas) after staining $(P=0.0025)$. Additional gastric adenomas were detected in 21 patients (42\%) with a median number of one adenoma (total 33, range 1-4) that had not been detected in the previous examination ( $\triangleright$ Fig. $\mathbf{1 b}$ and $\triangleright$ Table 4 ). All gastric adenomas were tubular with low-grade dysplasia.

Adenomas were classified to be either 1 to $4 \mathrm{~mm}, 5$ to $10 \mathrm{~mm}$ or $>10 \mathrm{~mm}$. After staining, there was no significant difference in the measured size of gastric adenomas $(P=0.4531)$.

The median amount of indigo carmine dye was $19.75 \mathrm{~mL}$ (range 10-35 mL) per patient. Inspection from the duodenal-jejunal junction to the gastric antral region took significantly longer using CE than the standard WLE examination (203 seconds ( \pm 125 seconds) vs 498 seonds ( \pm 423 seconds); $P<$ $0.0001)$. In the CE arm, staining was included in the withdrawal time.

No serious adverse events (AEs) were documented in the whole study. No documented AEs were associated with use of the study drug.

\section{Discussion}

Due to the significantly increased risk of duodenal carcinoma, regular endoscopic monitoring is essential in patients with FAP even after colectomy.
According to general consensus, the number and size of adenomas in the duodenum are decisive for prognostic estimation and thus for determination of monitoring intervals and therapeutic procedures. Therefore, improvement in endoscopic monitoring strategies continues to be of great importance. In this respect, CE in particular appears to be a promising approach.

Using either WLE or CE, a high ADR of over $86 \%$ was achieved in the upper gastrointestinal tract. Accordingly, no significant difference between CE and standard technique could be detected in analysis of the primary outcome. Therefore, our study was negative for the primary outcome measure.

However, we showed that after indigo carmine dye spraying, significantly more adenomas could be detected in all investigated sections of the gastrointestinal tract. In addition, CE tended to detect larger lesions in the duodenum. This together led to a significant increase in Spigelman stages in the duodenum.

Improved diagnostic performance of CE with increased detection of duodenal adenomas in patients with FAP has been demonstrated before $[13,15,17]$. However, the current study has several advantages compared to the previous studies. First, our study included the largest number of patients with FAP, with 50 individuals examined in our study compared to 10 patients in the study by Picasso et al. [17] and 19 patients with FAP analyzed by Hurley and co-workers [15]. Dekker et al. conducted a study in 43 patients with FAP [13]. Of them, however, only 26 patients had a confirmed APC mutation, whereas in our study, only carriers of a known pathogenic $A P C$ mutation took part.

Moreover, in the Dekker study, both endoscopic procedures were performed by the same endoscopist, which may have led to bias. In our study, both examinations were performed by independent endoscopists with the endoscopist performing CE being blinded to the prior endoscopic results.

Second, all previous studies on CE in patients with FAP demonstrated dye-spraying to increase the number of adenomas detected in the duodenum. However, in contrast to sporadic or hereditary non-polyposis colorectal cancer, ADR may not represent an appropriate measure reflective of cancer risk. Moreover, it remains unclear whether $C E$ also leads to a change in the Spigelman stage as currently available data are sparse and conflicting. Although recent data suggest that polyp size and highgrade dysplasia rather than number of adenomas or histology are associated with development of duodenal carcinomas [18], the Spigelman staging system still is widely accepted for guiding surveillance and therapeutic approaches.

In our study CE resulted in a higher classification in 16 of 50 patients (32\%), indicating the clinical relevance of our findings, although it has to be noted that the Spigelman staging system initially was validated using WLE endoscopy.

The observation that with CE duodenal lesions were classified to be larger than in WLE appears to be counterintuitive and is in contrast to previous studies. However, duodenal adenomas very often display a non-polypoid shape and polyp margins that are difficult to see. In this context, contrast enhancement may help better differentiate between adenomatous and non-adenomatous tissue, enabling more accurate measure- 
ment of polyp size. As a consequence, underestimation of polyp size rather than missing large polyps during WLE may explain our finding of larger polyps detected by CE. Of note, similar findings were reported in two previous colorectal CE studies, demonstrating a trend towards larger adenomas detected by CE $[19,20]$. It is important to note that detection of polyps larger than $10 \mathrm{~mm}$ leads to a change in clinical decision-making. The European Society for Gastrointestinal Endoscopy (ESGE) recommends performing EMR on all duodenal lesions larger than $10 \mathrm{~mm}$ [21]. In our study we detected significantly more adenomas larger than $10 \mathrm{~mm}$ using CE compared to WLE $(P=0.0391)$.

Third, our study is the first that also tested the diagnostic performance of CE in the gastric antral region. Recently, several reports described an alarming increase in incidence of gastric cancers (GC) in FAP $[6,22,23]$. Although the precursor lesion for gastric cancers in patients with FAP remains to be identified, Leone et al. demonstrated gastric adenomas to be more prevalent in patients with FAP with gastric cancer than in FAP control subjects [22]. In our study, indigo carmine dye-spraying enabled detection of additional gastric adenomas in 21 of 50 patients (42\%). A limitation of our study is the fact that we did not include the proximal stomach. Mankaney et al described an elevated gastric cancer risk in this region of the stomach where a polyposis is common in patients with FAP [6]. Use of CE might lead to better identification of high-grade dysplasia or at-risk polyps.

There are several other limitations to our study. Although use of CE led to a higher ADR compared to WLE (96 vs 86\%), this difference failed to reach statistical significance. In previous studies ADR using WLE was reported as being $30 \%$ to $65 \%[12,24]$, which is markedly lower than in our study. Thus, while the unexpectedly high ADR in our study highlights the endoscopists' experience and the quality of the examinations performed, it also caused our study to be underpowered to detect a significant difference in ADR between CE and WLE. A more ideal endpoint would have been to explore in how many patients use of CE would have led to different clinical management, such as EMR or shortening of surveillance interval. In our study, CE led to a significant difference in all these potential endpoints. In 16 patients a higher Spigelman stage led to a shorter surveillance interval. In nine patients adenoma size was $>10 \mathrm{~mm}$ using $\mathrm{CE}$, therefore indicating the need for EMR. Moreover, CE took significantly longer than WLE. Therefore, it cannot be unambiguously excluded that the higher ADR observed in CE may just reflect the longer duration of the endoscopic procedure. However, this seems rather unlikely as the increase in examination time was mainly attributable to the time needed for application of the dye and suctioning of the residual staining agent.

It should be noted that an older generation of endoscopes was used in our study. Current devices have much better image quality and offer the possibility of optical magnification, which together result in better definition and quality of mucosa visualization, thereby possibly enabling increased adenoma detection. Whether CE also offers an advantage with regard to ADR in this context needs to be evaluated in future studies. This also applies to virtual CE like blue laser imaging (BLI) as well as narrow-band imaging (NBI) endoscopy, which have been shown to enable better detection of (pre-)malignant lesions in the stomach [25].

Besides adenomas $>1 \mathrm{~cm}, \mathrm{CE}$ had the highest impact on detection of small adenomas. This resulted in an upgrade of the median Spigelman stage of 2 to 3 . This result might lead to overestimation of duodenal cancer risk due to the exceedingliy high number of small adenomas. Duodenal adenomas tend to be more indolent than colorectal adenomas. Therefore, detection of more small adenomas may result in overtreatment. This problem shows that the Spigelman classification should be revised.

\section{Conclusion}

Taken together, this is the largest study in proven APC-mutation positive patients with FAP to date analyzing the benefit of CE in the upper gastrointestinal tract and the first prospective study to evaluate the use of indigo carmine spray to improve ADR in the gastric antral region in patients with FAP. We demonstrated that CE significantly enhances ADR in patients with FAP, thereby resulting in an upgrade in the clinically relevant staging systems (Spigelman stage). Thus, our data demonstrate that CE is a useful tool for improved adenoma detection in patients with FAP.

Future studies are needed to investigate the potential longterm effects of this surveillance method on morbidity and mortality in polyposis patients.

\section{Acknowledgements}

The study was funded by a grant (O-107.0118) from the medical faculty of the University of Bonn (BONFOR). The funding body had no role in the design of the study and collection, analysis, and interpretation of data and in writing the manuscript. The authors thank Mrs. Melanie Neuhaus for her outstanding administrative assistance, the patient advocacy group (Familienhilfe Polyposis e. V.) and all the patients participating in this trial.

\section{Competing interests}

The authors declare that they have no conflict of interest.

\section{References}

[1] Aretz S, Uhlhaas S, Caspari R et al. Frequency and parental origin of de novo APC mutations in familial adenomatous polyposis. Euro J Human Genet 2003; 12: 52

[2] Leoz ML, Carballal S, Moreira L et al. The genetic basis of familial adenomatous polyposis and its implications for clinical practice and risk management. Appl Clin Genet 2015; 8: 95-107

[3] Bülow S, Björk J, Christensen IJ et al. Duodenal adenomatosis in familial adenomatous polyposis. Gut 2004; 53: 381-386

[4] Latchford AR, Neale KF, Spigelman AD et al. Features of duodenal cancer in patients with familial adenomatous polyposis. Clin Gastroenterol Hepatol 2009; 7: 659-663 
[5] Spigelman A, Talbot IC, Williams CB et al. Upper gastrointestinal cancer in patients with familial adenomatous polyposis. Lancet 1989; 334: 783-785

[6] Mankaney G, Leone P, Cruise M et al. Gastric cancer in FAP: a concerning rise in incidence. Familial Cancer 2017; 16: 371-376

[7] Syngal S, Brand RE, Church JM et al. ACG Clinical Guideline: Genetic Testing and Management of Hereditary Gastrointestinal Cancer Syndromes. Am J Gastroenterol 2015; 110: 223-262

[8] Vasen HF, Moslein G, Alonso A et al. Guidelines for the clinical management of familial adenomatous polyposis (FAP). Gut 2008; 57 : 704-713

[9] Heiskanen I, Kellokumpu I, Järvinen H. Management of duodenal adenomas in 98 patients with familial adenomatous polyposis. Endoscopy 1999; 31: 412-416

[10] Saurin J-C, Gutknecht C, Napoleon B et al. Surveillance of duodenal adenomas in familial adenomatous polyposis reveals high cumulative risk of advanced disease. J Clin Oncology 2004; 22: 493-498

[11] Groves C], Saunders BP, Spigelman AD et al. Duodenal cancer in patients with familial adenomatous polyposis (FAP): results of a 10 year prospective study. Gut 2002; 50: 636

[12] Björk J, Åkerbrant H, Iselius L et al. Periampullary adenomas and adenocarcinomas in familial adenomatous polyposis: Cumulative risks and APC gene mutations. Gastroenterology 2001; 121: 1127-1135

[13] Dekker E, Boparai KS, Poley JW et al. High resolution endoscopy and the additional value of $\mathrm{CE}$ in the evaluation of duodenal adenomatosis in patients with familial adenomatous polyposis. Endoscopy 2009; 41: 666-669

[14] Lopez-Ceron M, van den Broek FJ, Mathus-Vliegen EM et al. The role of high-resolution endoscopy and narrow-band imaging in the evaluation of upper gastrointestinal neoplasia in familial adenomatous polyposis. Gastrointest Endosc 2013; 77: 542-550

[15] Hurley JJ, Thomas LE, Walton S-J et al. The impact of CE for surveillance of the duodenum in patients with MUTYH-associated polyposis and familial adenomatous polyposis. Gastrointest Endosc 2018; 88: 665-673

[16] Schlemper RJ, Riddell RH, Kato Y et al. The Vienna classification of gastrointestinal epithelial neoplasia. Gut 2000; 47: 251-255

[17] Picasso M, Filiberti R, Blanchi S et al. The role of chromoendoscopy in the surveillance of the duodenum of patients with familial adenomatous polyposis. Digest Dis Sci 2007; 52: 1906-1909

[18] Thiruvengadam SS, Lopez R, O'Malley M et al. Spigelman stage IV duodenal polyposis does not precede most duodenal cancer cases in patients with familial adenomatous polyposis. Gastrointest Endosc 2019; 89: 345-354.e342

[19] Pohl J, Schneider A, Vogell H et al. Pancolonic CE with indigo carmine versus standard colonoscopy for detection of neoplastic lesions: a randomised two-centre trial. Gut 2011; 60: 485-490

[20] López-Vicente J, Rodríguez-Alcalde D, Hernández L et al. PanCE increases detection of polyps in patients with serrated polyposis syndrome. Clin Gastroenterol Hepatol 2019; 17: 2016-2023

[21] van Leerdam ME, Roos VH, van Hooft JE et al. Endoscopic management of polyposis syndromes: European Society of Gastrointestinal Endoscopy (ESGE) Guideline. Endoscopy 2019; 51: 877-895

[22] Leone PJ, Mankaney G, Sarvapelli S et al. Endoscopic and histologic features associated with gastric cancer in familial adenomatous polyposis. Gastrointest Endosc 2019; 89: 961-968

[23] Walton S-J, Frayling IM, Clark SK et al. Gastric tumours in FAP. Familial Cancer 2017; 16: 363-369

[24] Sourrouille I, Lefèvre JH, Shields C et al. Surveillance of duodenal polyposis in familial adenomatous polyposis: should the Spigelman score be modified? Dis Colon Rectum 2017; 60: 1137-1146

[25] Dohi O, Yagi N, Naito Y et al. Blue laser imaging-bright improves the real-time detection rate of early gastric cancer: a randomized controlled study. Gastrointest Endosc 2019; 89: 47-57 